\title{
Experimental and molecular dynamics study of gas flow characteristics in nanopores
}

\author{
LIU QiXin, JIANG PeiXue* \& XIANG Heng \\ Key Laboratory for Thermal Science and Power Engineering of Ministry of Education, Beijing Key Laboratory of $\mathrm{CO}_{2}$ Utilization and Reduction \\ Technology, Department of Thermal Engineering, Tsinghua University, Beijing 100084, China
}

Received September 5, 2011; accepted December 14, 2011

\begin{abstract}
Gas flow characteristics in nanopores were investigated experimentally and numerically using molecular dynamics (MD) simulations with an emphasis on the friction factor and gas viscosity. The results show that the viscosity and the friction factor in nanopores are much lower than those in macroscale channels. The actual viscosities obtained from the MD studies showed that the gas viscosity in nanopores is less than the macroscale viscosity because collisions between gas molecules are less frequent in high Knudsen number flows and there are more collisions with the wall. The MD simulations show that the velocity profile is composed of two parts, with a much steeper velocity gradient near the wall.
\end{abstract}

gas flow, nanopore, friction factor, molecular dynamics simulation

Citation: Liu Q X, Jiang P X, Xiang H. Experimental and molecular dynamics study of gas flow characteristics in nanopores. Chin Sci Bull, 2012, 57: 1488-1493, doi: 10.1007/s11434-012-5088-0

The rapid developments in nanotechnology require a deeper understanding of the gas flow characteristics in nanopores. All materials have unique properties on the nanometer scale and fluid flow in nanoscale spaces is different from that in macroscale spaces. The study of fluid flow characteristics in nanoscale spaces has become an important area of research [1-3]. A thorough understanding of the gas flow mechanisms and properties in nanoscale spaces is necessary for nanocatalysts, fuel cells, production of shale gas, cooling of nanomechanical-electrical systems (NMES), and theoretical developments in nanoscale fluid mechanics. For thermochemical reactions on the surfaces of nanocatalysts, an understanding of the gas flow characteristics in nanopores is critical for the understanding of nanocatalyst function and reaction speed control.

When the system size becomes comparable to the meanfree-path of the molecules, the assumption of fluid continuity is no longer appropriate. The Knudsen number $(K n)$ is defined as the ratio of the fluid mean-free-path, $\lambda$, to the system length, $L$ :

*Corresponding author (email: jiangpx@ @tsinghua.edu.cn)

$$
K n=\frac{\lambda}{L},
$$

where $\lambda=2 \mu / \rho \sqrt{\frac{8 k_{\mathrm{B}} T}{\pi m}}$ for an ideal gas. The Knudsen number can be used to divide gas flow regimes into four regions: $K n<0.001$ is the continuum region, $0.001<K n<$ 0.1 is the slip region in which the Navier-Stokes equations with the slip boundary condition are appropriate, $0.1<K n<$ 10 is the transition region, and $10<K n$ is the free molecular region. The debate still continues as to whether the hydrodynamics model based on the Navier-Stokes equations is appropriate for flows in the transition region and the free molecular region.

Majumder et al. [4] experimentally studied water flow characteristics through carbon nanotubes and found that liquid flow through a membrane composed of an array of aligned carbon nanotubes is four to five orders of magnitude faster than would be predicted from conventional fluid flow theory. Verwij et al. [5] and Holt et al. [6] experimentally studied the flow characteristics of gases and water in carbon nanotubes and found the flow characteristics differ greatly 
from Knudsen diffusion and Poiseuille flow, with fluxes much larger than that of Knudsen diffusion. Roy et al. [7,8] experimentally studied gas flow through $200 \mathrm{~nm}$ and $10 \mathrm{~nm}$ nanopores and found that the flow through $200 \mathrm{~nm}$ nanopores can be described by Knudsen diffusion; however, the measured flow rate through $10 \mathrm{~nm}$ nanopores was more than one magnitude larger than that predicted by Knudsen diffusion, and could be described by the Navier-Stokes equations with a slip boundary condition. Cooper et al. [9] also showed experimentally that the Navier-Stokes equations can describe the flow in $200 \mathrm{~nm}$ nanopores if the slip boundary condition is applied.

In addition to these experimental studies, molecular dynamics (MD) simulations have been used to study fluid flows in nanopores. Travis et al. [10-12] used non-equilibrium MD simulations to study Poiseuille flow between two plates several nanometers apart and found that when the distance between the two plates is larger than five times the mean molecular distance the velocity distribution, except near the plates, can be described by the Navier-Stokes equations. However, when the distance between the plates is less than five times the mean molecular distance, the velocity distribution deviates from a quadratic velocity distribution. Bhattacharya et al. [13] studied gas flow for a Knudsen number of 0.27 to show that the Navier-Stokes equations seem to be valid even for a Knudsen number of 0.27 . Xie et al. [14] studied Poiseuille flow of a mixture of gases in nanochannels using non-equilibrium MD simulations; the velocity distributions showed that the flow speeds increase as the hydrophobicity increases and the slip velocity of gas mixtures changes gradually from negative to positive. Markus et al. [15] examined molecular transport through membranes containing zeolite crystals and slit mesopores using MD simulations. Their results indicate that the presence of mesopores appreciably enhances the molecular flux through a membrane. Other researchers have used MD simulations to study problems related to nanoscale flows [16-18].

As the Knudsen number increases, gas molecules collide more frequently with the wall than with other gas molecules, so the effective viscosity of the gas should be smaller than that in a macroscale space. Veijola and Turowski [19] and Fichman and Hetsroni [20] performed theoretical analyses of the effective gas viscosity in nanospaces to illustrate that the effective viscosity of a gas in a nanospace is less than that in a large space. The MD simulation studies by Thomas et al. [21] showed that the viscosity of water decreases in carbon nanotubes with diameters as small as $1.66 \mathrm{~nm}$. To our knowledge, there have been no experimental studies of the effective viscosity of a gas in a nanospace. This paper presents experimental and MD studies of gas flow characteristics in nanopores, with an emphasis on the flow friction factor and the gas viscosity in the nanopores.

\section{Experimental study}

\subsection{Experimental system}

Experiments to study the gas flow through nanopores were conducted in a flow tube with a pressure drop across a nanopore membrane measured for controlled flow rates. The membranes were commercially available products. Three membranes were used in this study; their pore diameters and porosities were measured using the mercury intrusion method. The three membranes included alumina and polycarbonate materials. Figure 1 shows the pore diameter distributions in each membrane, with the most probable pore diameters in the three membranes being $226 \mathrm{~nm}, 110 \mathrm{~nm}$, and $61 \mathrm{~nm}$, with the nanopores aligned and running straight through the membrane. Their porosities were $13.4 \%, 12.8 \%$, and $31.4 \%$. Figure 2 shows scanning electron microscope images of the membrane surface and of a cross-section with pore diameters of $226 \mathrm{~nm}$. The pore lengths of the membranes are $6 \mu \mathrm{m}$ for both the $61 \mathrm{~nm}$ and $110 \mathrm{~nm}$ nanopores and $60 \mu \mathrm{m}$ for the $226 \mathrm{~nm}$ nanopores. The length-todiameter ratio is far more than 10-45 times the Reynolds number $(R e)$, so the flow is in the fully developed region.

A sketch of the experimental system is shown in Figure 3. Gas (air or argon) from a gas cylinder flowed through a gas filter, and then through a mass flow rate controller and mass flow meter, where the gas mass flow rate was measured,

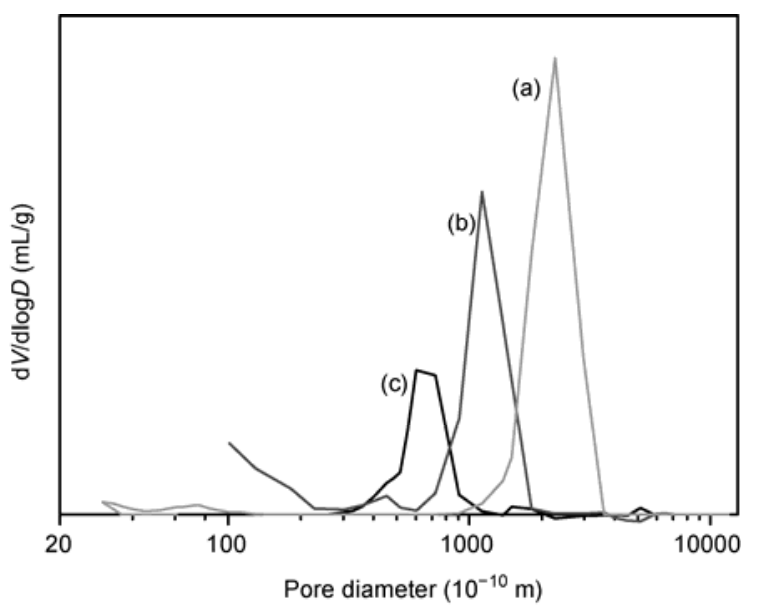

Figure 1 Pore diameter distribution in each membrane.
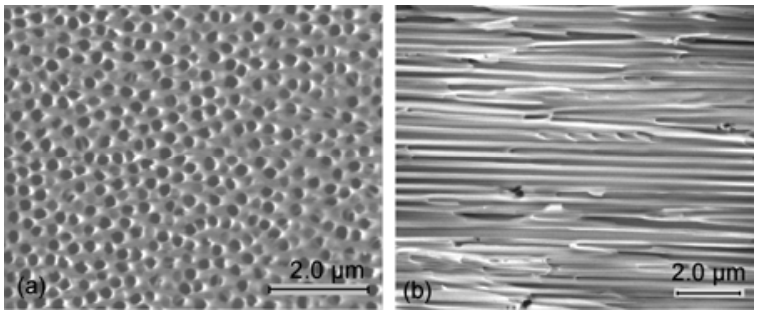

Figure 2 Scanning electron microscope images of a membrane surface (a) and a membrane cross-section (b). 


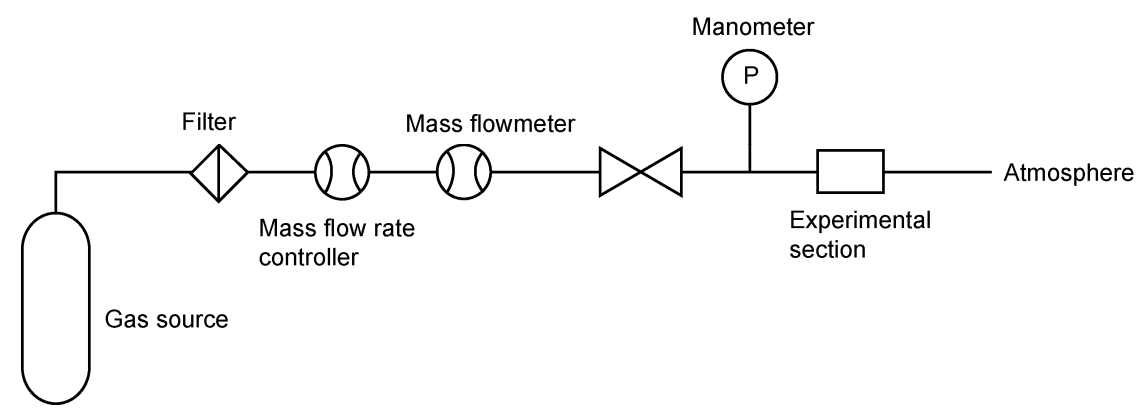

Figure 3 Experimental system.

and then through the experimental membrane. The pressure drop across the membrane was measured for a variety of flow rates. The inlet pressure was measured using pressure gauges with full ranges of $100,400,600$, and $1000 \mathrm{kPa}$. The air mass flow rate was measured using a gas mass flow meter (Sensirion model SFC4200), and the argon mass flow rate was measured using a gas mass flow meter produced by Alicat (Mass Flowmeter 20-1-00-5-5- KM2010).

\subsection{Friction factor}

The friction factor is an important flow characteristic and characterizes the flow through the nanopores. The average friction factor of a compressible fluid in a tube can be expressed as by Wang et al. [22]:

$$
\bar{f} \frac{L}{D}=\left.\left[\frac{1-M a^{2}}{\gamma M a^{2}}+\frac{(\gamma+1)}{2 \gamma} \ln \frac{(\gamma+1) M a^{2}}{2\left(1+\frac{\gamma-1}{2} M a^{2}\right)}\right]\right|_{0} ^{1},
$$

where $\gamma$ is the specific heat ratio, $M a$ is the Mach number, " 0 " stands for the beginning of the tube, and " 1 " stands for the end of the tube. For all the experiments, the second term on the right-hand side of eq. (2) was very small compared with the first term $(0.3 \%)$. The second term on the right-hand side of eq. (2) was therefore neglected. Using the definition of the Mach number, the gas friction factor can be

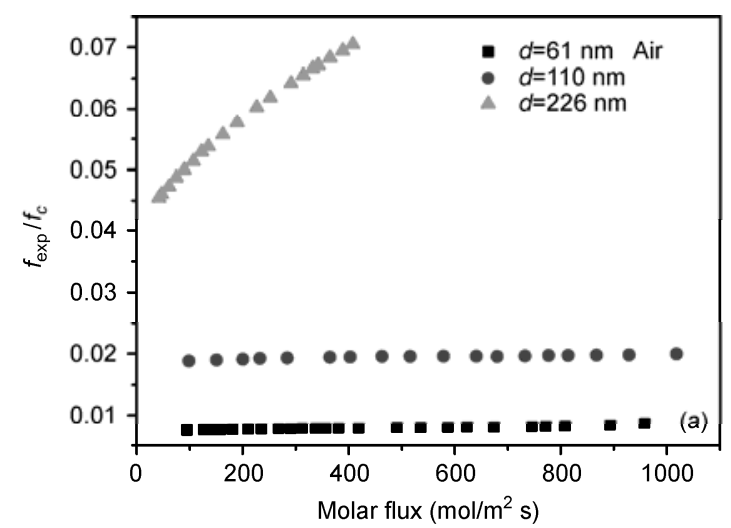

calculated from the experimental data as

$$
\bar{f}=\frac{D}{L} \frac{\rho_{f}}{(\rho u)^{2}} 2 \Delta P,
$$

where $\rho_{f}$ is the gas density corresponding to the mean pressure between the inlet and outlet, $D$ is the inner diameter of the nanopores, $L$ is the tube length, $\rho u$ is the mass flux, and $\Delta P$ is the gas pressure drop.

The accuracies of the pressure gauges were $0.1 \%$ of the full range. The maximum uncertainty in the pressure drop was therefore $\pm 1.0 \%$. According to the instructions, the accuracies of the mass flow meters were $\pm 0.8 \%$ of the actual mass flow rate plus $\pm 0.2 \%$ of the full range. The relative uncertainty of the mass flow rate was therefore $\pm 5.8 \%$. The maximum uncertainty of the membrane thickness was $\pm 2.0 \%$. The maximum uncertainties of the nanopore diameters and porosities were $\pm 2.0 \%$. A detailed uncertainty analysis showed that the maximum uncertainty of the friction factor was $\pm 12.7 \%$.

The theoretical value of the friction factor for fully developed laminar flow in round tubes is

$$
f_{c}=64 / R e \text {. }
$$

The ratios of the measured friction factors for argon and air flows in the nanopores to the theoretical values, shown in Figure 4, show that the measured friction factors in the nanopores are much less than the theoretical values. The

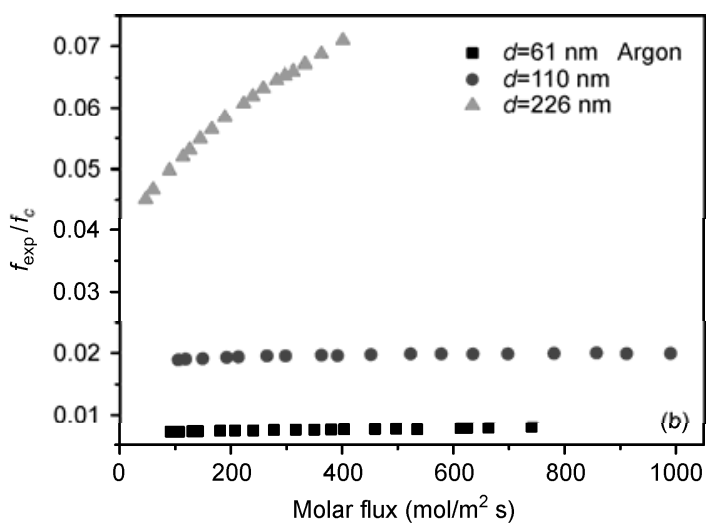

Figure 4 Ratios of measured to theoretical friction factors. 
Knudsen numbers for the $61 \mathrm{~nm}$ and $110 \mathrm{~nm}$ diameter nanopores varied from 0.41 to 1.0 and from 0.134 to 0.26 for the $226 \mathrm{~nm}$ diameter nanopores. For $0.1<K n<10$, the flow is in the transition region, but for $0.001<K n<0.1$, it is in the slip region; thus, the flows with the $61 \mathrm{~nm}$ and $110 \mathrm{~nm}$ nanopores were in the transition region, and the flows for the $226 \mathrm{~nm}$ diameter nanopores were also in the transition region but near the slip region. The data shows that the reduction in the friction factor depends on the Knudsen number, with the reduction in the friction factor for the $61 \mathrm{~nm}$ and $110 \mathrm{~nm}$ diameter nanopores being significantly larger than for the $226 \mathrm{~nm}$ nanopores. In addition, as the mass flux increases, the ratios of the measured friction factor to the theoretical value increase. For the $226 \mathrm{~nm}$ diameter nanopores, the change in this ratio with the flow rate is larger than for the other two nanopore sizes. With increasing pressure, the Knudsen number decreases and the measured friction factors approach the theoretical values.

\section{MD study}

\subsection{MD model}

MD simulations have become an important tool for analysis in nanoscale physics. The MD method is able to give details of the gas flow, such as the velocity profile in the nanopores, that cannot be measured experimentally. Here, the NEMD method is used to simulate the argon gas flow in the nanopores.

The physical model for the MD analysis is shown in Figure 5. The gas molecules move in nanopores made of fixed solid atoms. The gas and solid atoms are all assumed to be argon. Since only the gas flow is analyzed, the solid atoms are assumed to be stationary during the process. Argon gas is used because the L-J potential for argon is well known and can precisely describe the movements of the argon gas molecules, enabling qualitative comparison with the experimental results. The L-J potential is

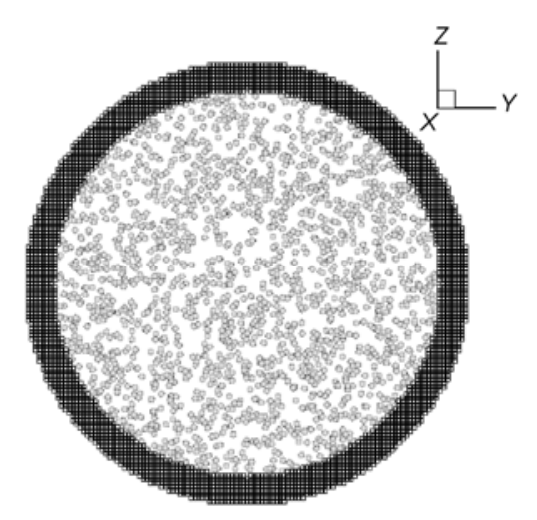

Figure 5 Cross-section of the particle locations in the tube used for the MD simulations.

$$
U(r)=4 \varepsilon\left[\left(\frac{\sigma}{r}\right)^{12}-\left(\frac{\sigma}{r}\right)^{6}\right]
$$

where the parameters for argon are $\varepsilon=1.67 \times 10^{-21} \mathrm{~J}$ and $\sigma$ $=3.405 \times 10^{-10} \mathrm{~m}$. The interactions between the gas and solid atoms are also described by eq. (5), but with $\varepsilon=2.82 \times$ $c \times 10^{-21} \mathrm{~J}$ and $\sigma=3.405 \times 10^{-10} \mathrm{~m}$, where $c$ represents the strength of the interactions between the gas molecules and the wall atoms. The gas temperature was controlled at $300 \mathrm{~K}$.

To simulate the gas flow in the nanopores resulting from a pressure difference between the two ends of the nanopore, a force was added in the flow direction, which is the $x$-direction, for every gas particle in the simulation domain. The value of the force added to each gas particle depended on the pressure difference and the nanopore radius as

$$
F_{\text {add }}=\frac{\Delta P \cdot \pi D^{2}}{4 N},
$$

where $\Delta P$ is the pressure difference between the two ends of the nanopore, $D$ is the nanopore diameter, and $N$ is the number of gas particles in the simulation domain. The mean gas pressure was set at $0.35 \mathrm{MPa}$.

\subsection{Predicted argon friction factors in nanopores}

The MD simulations can be regarded as computer experiments. Here, the MD results are used to calculate the friction factor for the argon flow in the nanopores. The friction factor was calculated from the flow flux and the pressure difference using eq. (3). The pressure difference between the two ends of the nanotube in the simulations was fixed at $0.2 \mathrm{MPa}$ in all cases. Table 1 lists the ratios of the argon flow friction factors in the nanopores predicted by the MD simulations to the theoretical macroscale values which show that the friction factors in the nanopores are much smaller than the macroscale theoretical values. The ratio of the predicted friction factor to the theoretical macroscale friction factor $\left(f_{\text {simu }} / f_{c}\right)$ increases with increasing pore diameter; this is similar to the experimental results shown in Figure 4. There are differences between the MD results and the experimental data because the solid material model in the MD simulations differs from the actual material used in the experiments.

Table 1 Ratios of the argon friction factors in the nanopores predicted by MD simulations to the macroscale values

\begin{tabular}{cl}
\hline Diameter $(\mathrm{nm})$ & $f_{\text {simu }} / f_{c}$ \\
\hline 12.7 & 0.036 \\
17.0 & 0.046 \\
21.3 & 0.058 \\
27.5 & 0.072 \\
50.9 & 0.18 \\
\hline
\end{tabular}




\subsection{Argon viscosity in nanopores}

The gas viscosity is defined as

$$
\mu=\frac{\tau}{\left.(\partial u / \partial r)\right|_{\text {wall }}} .
$$

This shows that the viscosity is defined based on the frictional force between the gas molecules and the wall and the velocity gradient at the wall. The frictional force between the gas molecules and the wall, $\tau$, was calculated from the MD simulation. The L-J unit velocity profile in terms of $U^{*}$ in the nanopores (Figure 6) shows an obvious slope change in the velocity profile, with a quadratic velocity profile far from the wall. If the quadratic velocity profile was elongated to the wall, the argon viscosity determined using eq. (7) would be $27.2 \%$ of the macroscale value. Figure 7 shows the velocity profile just for parts with radius larger than 9.9 $\mathrm{nm}$ of the $21.3 \mathrm{~nm}$ diameter nanopores. Here, the argon viscosity near the wall calculated from the MD simulations is only $1.1 \%$ of the macroscale viscosity. The results in Figures 6 and 7 show that the entire velocity profile consists

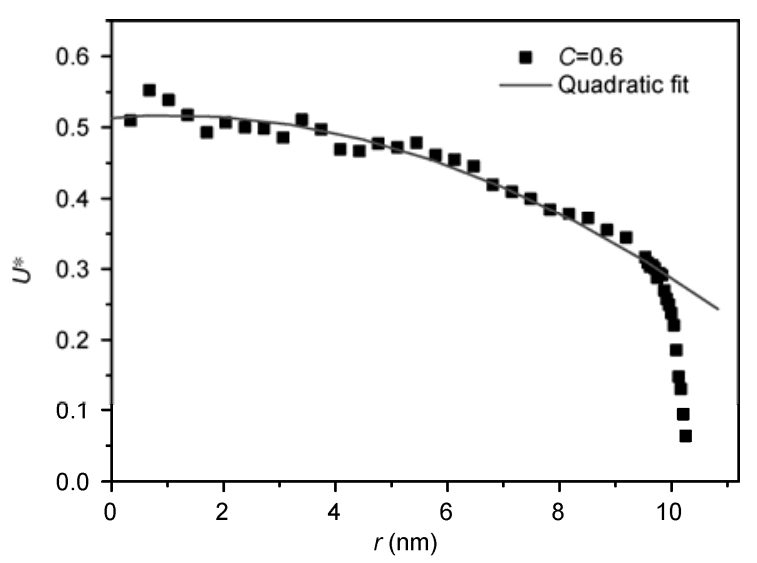

Figure 6 Entire velocity profile in the $21.3 \mathrm{~nm}$ nanopores.

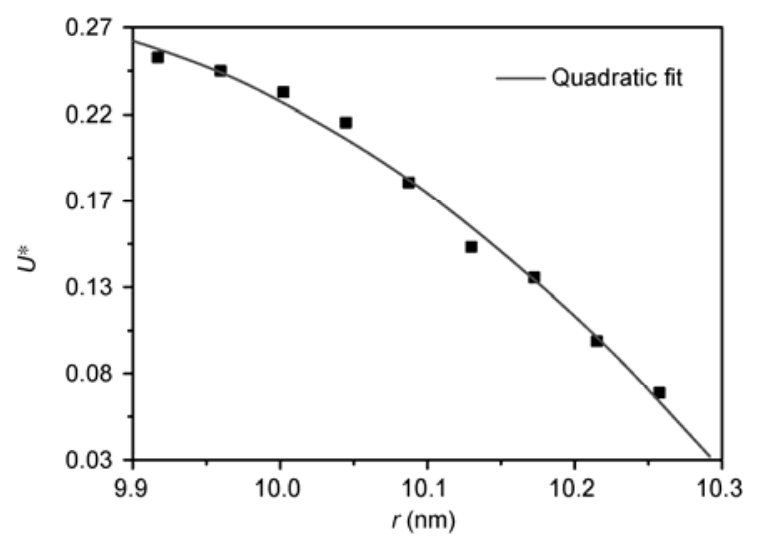

Figure 7 Predicted velocity profile near the wall for the $21.3 \mathrm{~nm}$ nanopores.

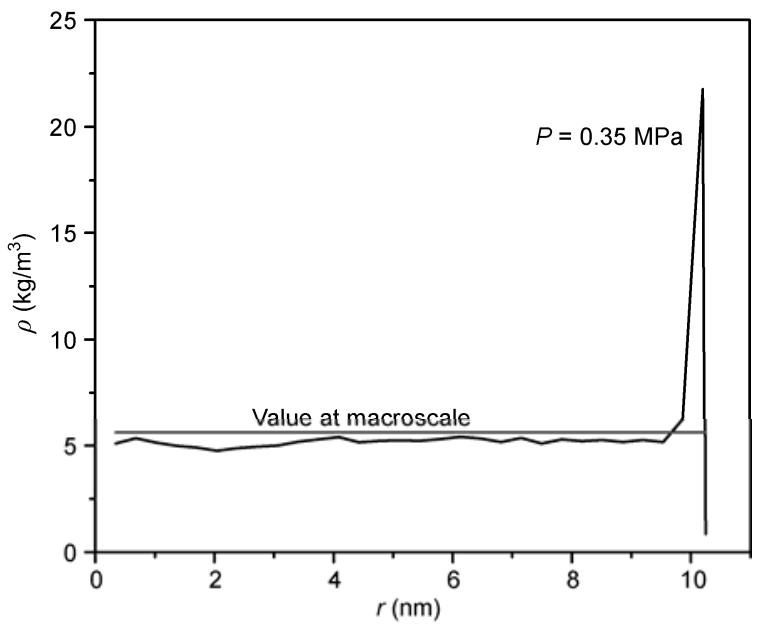

Figure 8 Argon gas density profile for the $21.3 \mathrm{~nm}$ nanopores.

of two parts with differences resulting from the different gas viscosities in the different regions since when the flow conditions such as the pressure difference are fixed the velocity profile depends only on the gas viscosity.

The collision frequency between the gas molecules is related to the gas molecule number density. Figure 8 shows the argon gas density profile from the MD simulations and the corresponding macroscale density at $300 \mathrm{~K}$. The argon gas density profile in the nanopores has one peak adjacent to the wall; this further illustrates that the ratio of the molecular collisions with the wall to collisions with other molecules increases near the wall, leading to a decrease in gas viscosity near the wall.

\section{Conclusions}

Experimental and MD studies of gas flows in nanopores show that the friction factors in the nanopores are much less than the macroscale friction factors. The actual viscosity obtained based on MD studies shows that the gas viscosity in nanopores is less than that at the macroscale because collisions between gas molecules are less frequent in high Knudsen number flows and there are more collisions with the wall. The MD simulations also show that the velocity profile is composed of two parts, whose differences are a result of the different gas viscosities in different parts of the nanopore.

We thank Prof. David Christopher for editing the English. The computations were supported by the THPCC. This work was supported by the National Natural Science Foundation of China (50676047 and 50736003).

1 Gravesen P, Branebjerg J, Jensen O S. Microfluidics-A review. J Micromech Microeng, 1993, 3: 168-182

2 Ho C M, Tai Y C. Micro-electro-mechanical-systems and fluid flows. Ann Rev Fluid Mech, 1998, 30: 579-612

3 Karniadakis G E, Beskok A. Micro Flows: Fundamentals and Simulation. New York: Springer, 2002 
4 Majumder M, Chopra N, Andrews R, et al. Nanoscale hydrodynamics: Enhanced flow in carbon tubes. Nature, 2005, 438: 44

5 Verwij H, Schillo M C, Li J. Fast mass transport through carbon nanotube membranes. Small, 2007, 3: 1996-2004

6 Holt J K, Park H G, Wang Y M. Fast mass transport through sub-2nanometer carbon nanotubes. Science, 2006, 312: 1034-1037

7 Roy S, Raju R, Chuang H F, et al. Modeling gas flow through microchannels and nanopores. J Appl Phys, 2003, 93: 4870-4879

8 Roy S, Cooper S M, Meyyappan M, et al. Single component gas transport through $10 \mathrm{~nm}$ pores: Experimental data and hydrodynamic prediction. J Membr Sci, 2005, 253: 209-215

9 Cooper S M, Cruden B A, Meyyappan M, et al. Gas transport characteristics through a carbon nanotube. Nano Lett, 2004, 4: 377-381

10 Travis K P, Gubbins K E. Poiseuille flow of Lennard-Jones fluids in narrow slit pores. J Chem Phys, 2000, 112: 1984-1994

11 Travis K P, Todd B D, Evans D J. Poiseuille flow of molecular fluids. Physica A, 1997, 240: 315-327

12 Travis K P, Todd B D, Evans D J. Departure from Navier-Stokes hydrodynamics in confined liquids. Phys Rev E, 1997, 55: 4288-4295

13 Bhattacharya D K, Lie G C. Molecular-dynamics simulations of nonequilibrium heat and momentum transport in very dilute gases. Phys Rev Lett, 1989, 62: 897-900

14 Xie H, Liu C, Liu B W. Molecular dynamics simulation of gas mix- ture flow in nanochannel. Acta Phys Chim Sin, 2009, 25: 994-998

15 Markus K, Sergey V. Molecular dynamics study of sorbate diffusion in a simple porous membrane containing microporous nanocrystals and mesopores. Chem Phys Lett, 2009, 479: 95-99

16 Feng J, Liao Q, Zhu X, et al. Molecular dynamics simulation of injection of polyethylene fluid in a variable cross-section nano-channel. Chin Sci Bull, 2011, 56: 1848-1856

17 Liu S Y, Yang X N, Qin Y. Molecular dynamics simulation of wetting behavior at $\mathrm{CO}_{2} /$ water/solid interfaces. Chin Sci Bull, 2010, 55: 2252-2257

18 Ren Y, Gao J, Ge W, et al. Molecular dynamics simulation of a single polymer in hydrophilic nano-slits. Chin Sci Bull, 2008, 53: 25992606

19 Veijola T, Turowski M. Compact damping models for laternally moving microstructures with gas-rarefaction effects. J Microelectromech Syst, 2001, 10: 263-273

20 Fichman M, Hetsroni G. Viscosity and slip velocity in gas flow in microchannels. Phys Fluids, 2005, 17: 123102

21 Thomas J A, McGaughey A J H, Kuter-Arnebeck O. Pressure-driven water flow through carbon nanotubes: Insights from molecular dynamics simulation. Int J Therm Sci, 2010, 49: 281-289

22 Wang B G, Liu S Y, Huang W G. Gas Dynamics. Beijing: Beijing Institute of Technology Press, 2005

Open Access This article is distributed under the terms of the Creative Commons Attribution License which permits any use, distribution, and reproduction in any medium, provided the original author(s) and source are credited. 